\title{
胆固醇代谢与肿瘤
}

\author{
孟 颖 ${ }^{1,2}$, 王启扉 ${ }^{1,2}$, 吕志民 ${ }^{1,2}$ \\ 1. 浙江大学医学院附属第一医院肝胆胰外科浙江省胰腺疾病重点实验室, 浙江杭州 310003 \\ 2. 浙江大学医学院转化医学研究院, 浙江杭州 310029
}

[ 摘 要] 胆固醇是哺乳动物体内重要的脂质, 是必不可少的膜结构成分。胆固醇 稳态平衡对维持细胞和机体的生命活动至关重要,机体主要通过内源胆固醇的生 物合成和外源胆固醇的摄取来获得胆固醇以维持自身稳态平衡。肿瘤细胞中胆固 醇代谢异常活跃, 导致其增殖、存活、侵袭、转移以及对肿瘤微环境的适应能力 增强。靶向胆固醇合成、降低血浆胆固醇水平和胆固醇酯化的治疗策略将为肿瘤 患者的治疗带来新的希望。本文总结了胆固醇代谢调节及其在肿瘤发生发展中的 研究进展, 并讨论了目前千扰胆固醇代谢的肿瘤治疗新方法。

[关键词] 胆固醇; 代谢; 肿瘤; 治疗; 综述

[中图分类号 ] R73 [文献标志码 ] A

\section{Cholesterol metabolism and tumor}

MENG Ying ${ }^{1,2}$, WANG Qifei ${ }^{1,2}$, LYU Zhimin ${ }^{1,2}$ (1. Zhejiang Provincial Key Laboratory of Pancreatic Disease, Department of Hepatobiliary and Pancreatic Surgery, the First Affiliated Hospital, Zhejiang University School of Medicine, Hangzhou 310003, China; 2. Institute of Translational Medicine, Zhejiang University School of Medicine, Hangzhou 310029, China )

Corresponding author:LYU Zhimin,E-mail:zhiminlu@zju.edu.cn,https://orcid.org/ 0000-0002-2859-2736

\begin{abstract}
[Abstract ] Cholesterol is an important lipid in the body of mammals and an essential component of membrane structures. Cholesterol homeostasis is critical for the maintenance of cellular and body activities, and is mainly regulated by the balance of de novo cholesterol biosynthesis and the exogenous cholesterol uptake. Aberrantly regulated cholesterol metabolism promotes tumor cell proliferation, survival, invasion and metastasis, and their adaptability into the tumor microenvironment. Therefore,targeting cholesterol biosynthesis and reduction of plasma cholesterol levels and cholesterol
\end{abstract}

\section{收稿日期:2020-11-25 接受日期:2020-12-31}

基金项目:科技部重点研发计划(2020YFA0803300); 浙江省引进培育领军型创新创业团队(2019R01001)

第一作者:孟 颖, 博士后, 主要从事胆固醇代谢及肿瘤发生发展相关研究; E-mail:ymeng@zju. edu. cn; https://orcid. org/0000-0002-1165-9009

通信作者:吕志民,教授,博士生导师,主要从事肿瘤代谢研究; E-mail:zhiminlu@zju. edu. cn; https://orcid. org/00000002-2859-2736 
esterification will provide new strategies for cancer treatment. This review summarizes the current understanding in cholesterol homeostasis regulation and its function in the occurence and development of cancer, as well as current metabolism-targeted cancer treatments.

[Key words] Cholesterol; Metabolism; Tumor; Therapy ; Review

[J Zhejiang Univ (Med Sci), 2021, 50(1): 23-31. ]

[缩略语] 3-着基-3-甲基戊二酸单酰辅酶 A 还原酶(3-hydroxy-3-methylglutaryl coenzyme A reductase,HMGCR); C1 型尼曼-匹克样蛋白 1(Niemann-Pick type C1 like 1, NPC1L1); 低密度脂蛋白 (low-density lipoprotein, LDL); 低密度脂蛋白-胆固醇(LDLcholesterol, LDL-C); 高密度脂蛋白 (high-density lipoprotein, HDL); 高密度脂蛋白-胆 固醇(HDL-cholesterol, HDL-C); B 类 I 型清道夫受体(scavenger receptor class B type 1,SR-B1); 固醇调节元件结合蛋白 2(sterol-regulatory element binding protein 2, $\mathrm{SREBP} 2$ ); 核 SREBP2(nuclear SREBP2,nSREBP2); 酰基辅酶 A : 胆固醇酰基转移酶 1 (acyl-coenzyme A:cholesterolacyltransferase 1,ACAT-1); 表皮生长因子受体(epidermal growth factor receptor, EGFR); 磷脂酰肌醇3-激酶 (phosphoinositide 3-kinase,PI3K); 蛋 白激酶 B (protein kinase B, Akt); 肝脏X 受体(liver X receptor, LXR); 前蛋白转化酶枯 草杆菌蛋白酶/kexin 9 型 (proprotein convertase subtilisin/kexin type 9,PCSK9); 胆固醇 酯转运蛋白 (cholesteryl ester transfer protein, CETP); CCAAT/增强子结合蛋白同源蛋 白 (CCAAT/enhancer-binding protein homologous protein, CHOP); 丝裂原活化蛋白激酶 (mitogen-activated protein kinase, MAPK)

胆固醇是真核生物细胞膜必不可少的结构成 分, 决定着细胞膜的生理特性。胆固醇可以由机 体内源合成, 也可通过外源摄取获得, 其代谢紊乱 与心脑血管疾病、糖尿病等多种疾病相关 ${ }^{[1-2]}$ 。研 究表明, 肿瘤发生发展过程中存在胆固醇代谢改 变, 主要表现为细胞内胆固醇水平上调, 代谢产物 异常堆积 ${ }^{[3]}$ 。寻找靶向胆固醇代谢途径的药物可 能为肿瘤患者的治疗带来新的希望。本文总结了 胆固醇代谢调节及其在肿瘤发生发展中的研究进 展, 并讨论了干扰胆固醇代谢的肿瘤治疗新 方法。

\section{1 胆固醇代谢及稳态平衡}

体内获取胆固醇以维持稳态平衡的方式主要 有两种, 一种是内源胆固醇的生物合成, 另一种是 外源胆固醇的摄取。

\section{1 内源胆固醇生物合成}

胆固醇的生物合成主要发生在肝脏, 细胞内 的内质网是其主要场所。该过程通过近 30 步酶 促反应(包括甲差圣戊酸中的途径) 将乙酰辅酶 $\mathrm{A}$ 转 化为胆固醇分子, 内质网跨膜蛋白 HMGCR 和角鲨
烯单加氧酶为该过程的限速酶(图 1)。HMGCR 是 定位于内质网的糖蛋白,可将 $\beta$-羟基- $\beta$-甲成二 酸单酰辅酶 $\mathrm{A}$ 还原为甲羟戊酸 ${ }^{[4]}$ 。HMGCR 在转 录、转录后、翻译和翻译后水平均受到调控。角鉴 烯单加氧酶(由 $S Q L E$ 编码) 是HMGCR 下游的另一 种胆固醇生物合成的限速酶, 可将非甾醇中间角 鲨烯转化为 2,3 -氧化角鲨烯。

\section{2 外源胆固醇摄取}

膳食胆固醇由肠上皮细胞膜上的 NPC1L1 吸 收获得,并进一步被ACAT(也称固醇 $\mathrm{O}$-酰基转移 酶)酯化, 以乳糜微粒的形式被肝脏吸收 ${ }^{[5]}$ 。肝脏 作为主要的胆固醇合成器官, 可将内源性合成和 外源性摄取的胆固醇以极低密度脂蛋白的形式输 送到血液中, 并进一步加工成 LDL 供外周细胞上 的 LDL 受体摄取。同时, 外周组织中多余的胆固 醇也会以 HDL-C 的形式被逆转运到肝脏进行再循 环或以胆汁酸的形式被排泄 ${ }^{[6]}$ 。

对于细胞而言,除少部分合成于内质网的胆 固醇外, 细胞内所需的大部分胆固醇主要来源于 受体介导的外源胆固醇的摄取 (图 2) ${ }^{[7]}$,包括胞吞 途径 ${ }^{[8]}$ 得到的 LDL-C 和选择性摄取途径 ${ }^{[9]}$ 得到 


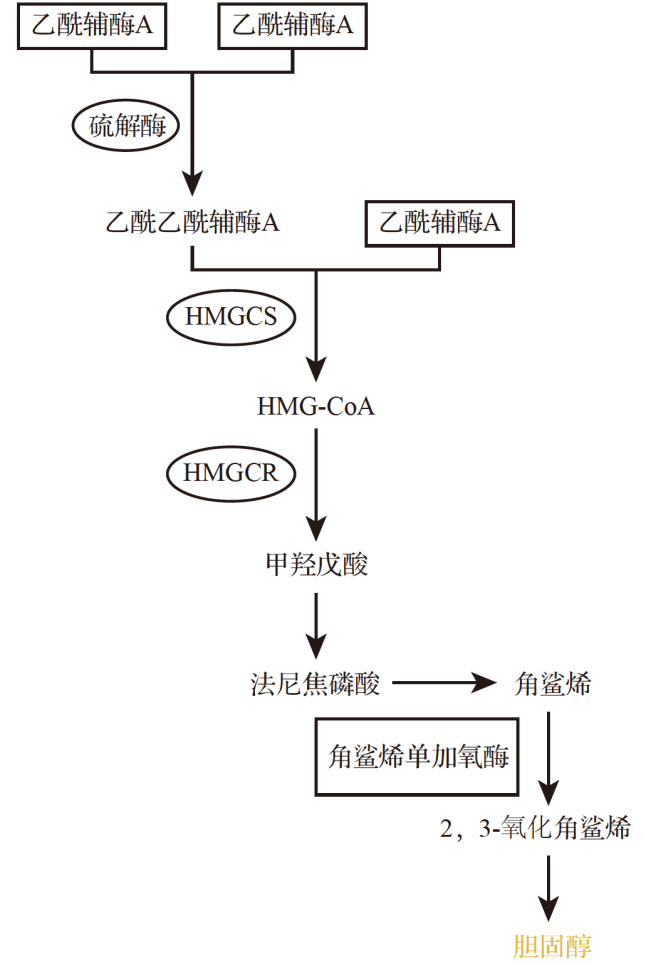

胆固醇的生物合成始于两分子的乙酰辅酶 $\mathrm{A}$, 后者在硫解酶 的作用下形成乙酰乙酰辅酶 $A$, 再通过 $\beta$-差基- $\beta$-甲戍二酸单酰 辅酶 $\mathrm{A}$ 合酶 (HMGCS) 与第三分子的乙酰辅酶 $\mathrm{A}$ 缩合形成 $\beta$-差基$\beta$-甲戊二酸单酰辅酶 $\mathrm{A}(\mathrm{HMG}-\mathrm{CoA})$; 3 -差基-3-甲基戊二酸单酰辅 酶 A 还原酶 (HMGCR) 将 HMG-CoA 还原成甲羟戊酸, 甲羟戊酸进 一步转化为法尼焦磷酸, 法尼焦磷酸转化成角滥烯并被角渻烯单 加氧酶氧化生成 2,3 -氧化角鲨烯,进而合成胆固醇.

图 1 胆固醇的生物合成

Figure 1 Cholesterol biosynthesis

的 HDL-C, 这两种途径共同调控了细胞内胆固醇 的稳态平衡。其中, 胞吞途径由 LDL受体介导, 是 经典的胆固醇摄取途径。LDL 颗粒与 LDL受体在 细胞膜结合后,通过内化最终到达溶酶体, 经酸性 脂肪酶水解释放游离胆固醇 ${ }^{[10]}$ 。随后, 游离胆固 醇被转运至溶酶体膜, 并进一步到达内质网进行 传感、转运或酯化 ${ }^{[11-12]}$, 同时也会被转运到线粒 体、过氧化物酶体以及细胞膜等其他细胞器 ${ }^{[7]}$ 。 选择性胆固醇摄取途径主要是 HDL 来源胆固醇的 摄取, 该途径不依赖于胞吞的发生。目前对选择 性胆固醇摄取的研究不多, 其功能也很容易被忽 略, 肝脏、肾上腺和卵巢等组织可优先通过 HDL受 体 SR-B1 从 HDL 中摄取胆固醇 ${ }^{[9]}$ 。HDL 颗粒与 细胞膜上的 SR-B1 结合后, 不需要像 LDL一样通 过胞吞途径到达溶酶体被降解 ${ }^{[13-14]}$, HDL 来源的 胆固醇和胆固醇酯通过 SR-B1 的疏水通道被摄取
到细胞膜 ${ }^{[15]}$, 并进一步转运到内质网等细胞器, 该过程的具体机制还有待进一步阐明。

\section{3 胆固醇的稳态平衡}

胆固醇的稳态平衡受到精密调控, 以避免游 离胆固醇含量过高引起细胞毒性 ${ }^{[16]}$ 。胆固醇的 合成和摄取相关基因主要由 SREBP2 转录调控 ${ }^{[17]}$ 。 当内质网膜胆固醇水平降低时, 位于内质网的转 录因子SREBP2 会迅速响应, 从内质网释放到达高 尔基体, 经历加工剪切形成活性的 nSREBP2进人 细胞核,进而上调胆固醇合成的限速酶 HMGCR和 胆固醇摄取相关 LDL受体表达, 从而促进胆固醇 的生物合成和外源摄取; 而当细胞内胆固醇累积 时则不会激活 SREBP2 通路,过量的胆固醇可在 ACAT-1 的作用下生成胆固醇酯,储存于脂滴; 也 可在胆固醇羟化酶的作用下生成羟胆固醇或环氧 化酶的催化下生成环氧胆固醇, 还可转化为维生 素 D、类固醇激素等物质 ${ }^{[18-19]}$ 。

\section{2 胆固醇代谢与肿瘤的发生发展}

\section{1 肿瘤细胞胆固醇代谢异常}

胆固醇可促进细胞增殖、迁移和侵袭, 在肿瘤 的发生发展中发挥重要作用。肿瘤细胞中胆固醇 代谢调控异常主要表现为胆固醇合成水平上调、 胆固醇摄取增加, 大量代谢产物异常堆积, 导致肿 瘤细胞增殖、存活、侵袭、转移以及对肿瘤微环境 的适应能力增强, 从而促进肿瘤的发生发展 ${ }^{\left[{ }^{[}\right]}$。

研究表明,多种与胆固醇合成相关的基因在 肿瘤组织中活性增加, 如胆固醇生物合成限速酶 HMGCR 和角鲨烯单加氧酶。HMGCR 的表达在多 种肿瘤中上调,包括胃癌、胶质瘤和前列腺癌 ${ }^{[20-22] 。}$

HMGCR 过表达促进了这些肿瘤细胞的生长和迁 移, 而抑制 HMGCR 则可抑制肿瘤的发生发展, 靶 向抑制 HMGCR 的表达已被用于治疗实体癌、血液 癌和具有耐药性的肿瘤 ${ }^{[23-25]}$ 。编码角鲨烯单加氧 酶的 SQLE基因座也在多种肿瘤中拷贝数增加, 其 在非酒精性脂肪肝引起的肝细胞癌中过量表达, 并且与胰腺癌的放射线耐受以及乳腺癌、前列腺 癌、结直肠癌和鳞状上皮癌的发展或患者预后不 良相关 ${ }^{[26-28]}$ 。

除了胆固醇合成相关蛋白高表达外,介导胆 固醇摄取的 LDL受体表达在多种肿瘤细胞中也呈 上调趋势。研究表明, LDL 受体表达与小细胞肺 癌患者的不良临床预后正相关 ${ }^{[29]}$ 。LDL受体高表 


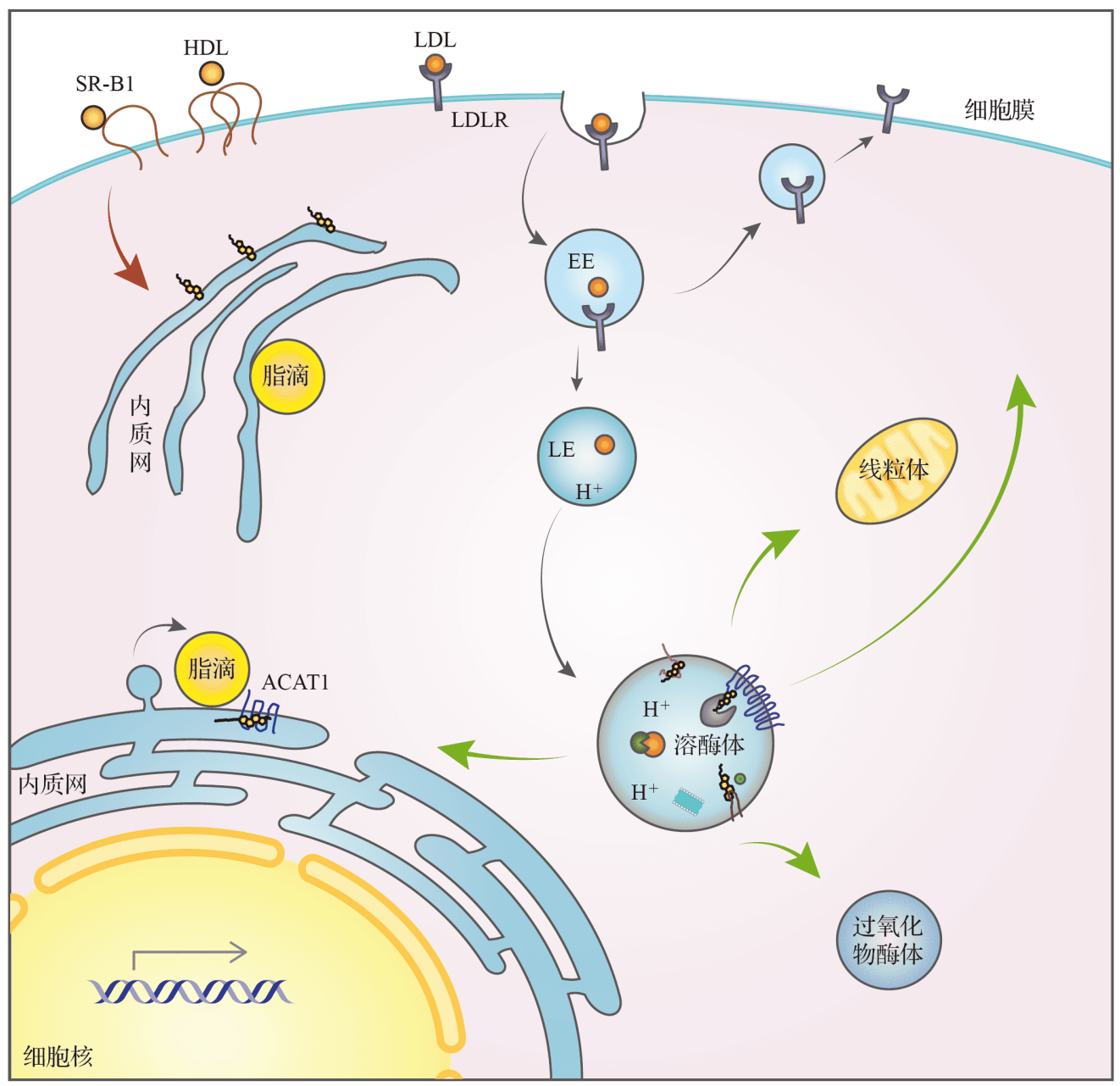

低密度脂蛋白 $(\mathrm{LDL})$ 颗粒与 LDL受体 (LDLR) 在细胞膜结合后, LDL-LDLR 复合物首先通过内吞作用到达早期内体 (EE) ; 在酸性 的 EE 中, LDLR 与 LDL 颗粒分离返回至细胞膜循环利用, 而 LDL 颗粒则进一步被转运至晚期内体 (LE) 并最终到达溶酶体; 在溶酶体中, LDL 来源的胆固醇酯被酸性脂肪酶水解,释放游离胆固醇, 随后游离胆固醇被转运至溶酶体膜, 并最终到达内质网进行传感、转运或酯化后 储存于脂滴, 同时也会被转运到线粒体、过氧化物酶体以及细胞膜等其他细胞器. 高密度脂蛋白 (HDL)颗粒与细胞膜上的 B 类型清道夫受 体 (SR-B1) 结合后, SR-B1 通过自身的疏水通道将 HDL 来源的胆固醇和胆固醇酯摄取到细胞质膜, 并进一步转运到内质网等细胞器. ACAT-1 : 酰基辅酶 A : 胆固醇酰基转移酶 1 .

图 2 受体介导的外源胆固醇摄取

Figure 2 Receptor-mediated cholesterol uptake

达与乳腺癌患者无复发存活率降低相关, 而在人 表皮生长因子受体 2 过表达的乳腺癌细胞中, 沉 默 LDL 受体则可以延缓高脂血症小鼠肿瘤的生 长 $^{[30]}$ 。同样, 胰腺导管腺癌患者 LDL受体表达升 高与复发率增加相关, 而肤腺肿瘤细胞中 LDL受 体缺失可显著减少胆固醇摄取,抑制肿瘤细胞增 殖 ${ }^{[31]}$ 。此外, 在胶质瘤细胞中, LDL受体表达高于 邻近的正常脑组织,并且与 EGFR 激活和 nSREBP1 表达相关 ${ }^{[32]}$ 。前列腺癌细胞中肿瘤抑制因子磷 酸酯酶与张力蛋白同源物缺失和 PI $3 \mathrm{~K} / \mathrm{AKT}$ 通路 激活可显著增加肿瘤生长所需的外源 LDL 的摄 取 ${ }^{[33]}$ 。除了 LDL受体表达水平升高外,一些肿瘤
细胞中 HDL 受体 SR-B1 的表达也会升高, 如胶质 母细胞瘤中过表达的 SR-B1 可促进肿瘤侵袭 ${ }^{[34]}$ 。 这些研究表明,异常表达的 LDL受体和 SR-B1 对 胆固醇的摄取在某些类型肿瘤细胞的增殖和侵袭 中扮演着重要角色。

\section{2 肿瘤细胞胆固醇代谢调控机制}

2.2.1 调节SREBP 活性对胆固醇代谢的影响 SREBP 激活在肿瘤细胞胆固醇代谢异常中起核心 作用。通常情况下,SREBP 由 INSIG 蛋白、SREBP 剪切激活蛋白和内质网胆固醇抑制。在肿瘤细胞 中, 过度活化的 PI3K/AKT 信号通路以及 $\mathrm{p} 53$ 介导的 信号通路可调控SREBP 的活性。PI3K/AKT信号通 
路通过哺乳动物雷帕需素靶蛋白复合物 1 依赖 的方式激活SREBP, 从而促进胆固醇的生物合成 和摄取 ${ }^{[32]}$ 。研究表明, 在胶质瘤细胞中, EGFR VIII突变体/PI3K 可激活 SREBP1, 从而上调肿瘤细 胞中 LDL受体表达, 促进外源胆固醇的摄取; 而 p53则通过在mRNA水平抑制SREBP, 调节其活性, 从而抑制胆固醇的合成和摄取 ${ }^{[35]}$ 。除此之外, 最 新研究表明,在肝癌细胞中, 激活的 AKT 可磷酸 化细胞质中的糖异生限速酶磷酸烯醇丙酮酸羧化 激酶 1 , 使其易位到内质网并作为蛋白激酶磷酸化 INSIG蛋白。这种磷酸化作用减少了固醇与 INSIG 的结合, 并破坏了 INSIG 蛋白与 SREBP 剪切激活 蛋白之间的相互作用, 从而激活 SREBP 以及下游 脂质合成和摄取相关基因的转录表达, 促进肿瘤 的发生发展 ${ }^{[36]}$ 。

2.2.2 ACAT-1 对胆固醇代谢的影响 胆固醇酯 的形成是防止游离胆固醇积累的重要方式。内质 网跨膜蛋白 ACAT-1 可将过量的胆固醇酯化生成 胆固醇酯并储存于脂滴中。研究表明, 乳腺癌、胰 腺癌、胶质母细胞瘤等肿瘤细胞中 ACAT-1 的表达 和活性均升高, 胆固醇酯的含量上调 ${ }^{[37]}$ 。过量胆 固醇酯的生成会激活 SREBP1, 从而促进肿瘤转 移; 而抑制 ACAT-1 可通过阻断 SREBP1 调控的基 因表达, 抑制胶质母细胞瘤的生长和前列腺癌细 胞的侵袭性 ${ }^{[33]}$ 。

2. 2.3 LXR 对胆固醇代谢的影响 LXR 被胆固 醇激活后可与 SREBP 拮抗参与体内胆固醇稳态的 调控 ${ }^{[38]}$ 。LXR 在肿瘤细胞脂质代谢中的作用因 其调控多个下游基因的表达而变得复杂。LXR 可 通过增加胆固醇外排和减少胆固醇摄取降低细胞 内胆固醇水平。前者由 LXR 通过调节载脂蛋 白 $\mathrm{E}$ 及其转运蛋白三磷酸腺苷结合盒转运体 A1 和 G1 的表达引起, 后者通过促进 LDL受体降解介 导 ${ }^{[39-40]}$ 。研究表明,LXR 激动剂或合成配体可抑 制不同类型肿瘤(如胶质瘤和乳腺癌)的增殖和生

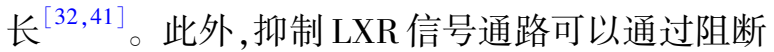
糖酵解和脂肪酸生物合成酶的表达来诱导多种 肿瘤细胞凋亡 ${ }^{[42]}$ 。LXR 的激活还可抑制免疫系 统 ${ }^{[43-44]}$, 通过降低细胞内胆固醇的含量来抑制淋 巴细胞的增殖, 从而影响体内免疫系统的稳态和 免疫应答。鉴于 LXR 激活也可诱导细胞周期调节 因子和参与免疫细胞功能相关基因的表达, 因此 在完整的肿瘤免疫微环境中探究 LXR 激动剂或拮
抗剂在抑制肿瘤中的作用具有重要意义。

\section{3 靶向胆固醇代谢在肿瘤治疗中的应用}

目前,在不同水平上干预胆固醇代谢的治疗 药物的相关研究已经取得了很大的进展。靶向胆 固醇合成相关代谢酶、脂蛋白介导的胆固醇摄取 以及胆固醇储存途径可能成为潜在的预防或延缓 相关肿瘤进展的有效途径。

\section{1 靶向胆固醇合成的治疗}

肿瘤细胞胆固醇合成途径有多种潜在的抑制 性靶点 ${ }^{[45]}$, 针对胆固醇合成相关蛋白的治疗药物 已开展临床研究。其中,抑制甲羟戈酸途径限速 酶 HMGCR 的他汀类药物可通过破坏细胞膜的完 整性以及细胞稳态的维持、影响细胞存活相关蛋 白的活性等多种途径发挥抗肿瘤作用 ${ }^{[46-49]}$ 。他汀 类药物是最常见的降胆固醇药物, 同时也是肿瘤 尤其是乳腺癌临床研究中使用最广泛的胆固醇代 谢靶向药物。

不同他汀类药物的特殊化学性质决定了其机 制特性。普伐他汀是一种亲水性他汀类药物, 被 一种仅在肝脏中表达的不依赖钠的有机阴离子转 运多肽1B1 选择性吸收。而亲脂性HMGCR抑制剂 辛伐他汀是一种通过其他机制进人细胞的疏水性 他汀类药物。辛伐他汀治疗可降低甲羟戈酸途径 产生的香叶基香叶基焦磷酸(蛋白异戊二烯化所 必需) 以及抗原呈递细胞中小 GTP酶Rab5的异戊 二烯化水平, 从而阻滞胞内体成熟, 延长抗原保留 时间，增强抗原提呈和 $\mathrm{T}$ 细胞激活，最终增强抗肿 瘤免疫力。此外,辛伐他汀可强有力地增强肿瘤 疫苗效力并与抗程序性死亡受体 1 抗体协同作用 进行肿瘤治疗 ${ }^{[50]}$ 。

回顾性研究表明,他汀类药物治疗可延长多 发性骨髓瘤、结直肠癌和转移性胰腺癌患者接受 一线化疗药物联合治疗的存活期 ${ }^{[51-53]}$ 。值得注意 的是, II 期临床试验结果显示, 辛伐他汀与EGFR 抑制剂吉非替尼 (gefitinib)联合使用比单纯使用 EGFR抑制剂治疗非小细胞肺癌抗肿瘤效果更 优 ${ }^{[54-55]}$ 。有研究表明, 亲脂性他汀类药物更容易 进人肝外细胞, 而亲水性他汀类药物对肝脏的选 择性更高 ${ }^{[56]}$ 。此外, 临床数据也表明他汀类药物 的抗肿瘤作用是剂量和时间依赖的 ${ }^{[57]}$ 。因此, 寻 找用于患者分层的预测性生物标志物将为他汀类 药物辅助治疗提供更准确的评估。 


\section{2 靶向血浆胆固醇水平的治疗}

研究表明,依折麦布 (Ezetimibe)可作用于NPC$1 \mathrm{~L} 1$, 显著降低肠道对胆固醇的吸收, 使血浆胆固 醇水平下降或增强血浆清除胆固醇的能力 ${ }^{[58]}$ 。 此外, LDL 受体表达受到 PCSK9 的调控, 抑制 PCSK9 的药物可导致肝脏 LDL 受体丰度增加, 有 效降低血浆中 LDL-C 水平 ${ }^{[59]}$, 这是一种通过限制 外源性脂质的量以抑制肿瘤增殖的策略。近期研 究表明, 提高 LDL-C 水平的PCSK9 基因突变与乳 腺癌的高风险相关, 而降低 LDL-C 水平的PCSK 9 基因突变则可显著降低雌激素受体阳性乳腺癌的 风险 ${ }^{[60]}$ 。近年研究也逐渐出现了一些基于改 变 HDL 水平的治疗策略, 以预防肿瘤的发展。目 前, 已有实验模型和大型临床试验对 CETP抑制剂 和其他药物 (如贝特类和烟酸) 提高血浆 HDL-C 水 平的效果进行评估 (例如对内分泌相关癌症的研 究 $)^{[61]}$ 。其中 CETP 抑制剂升高 HDL-C 水平的作 用最强。CETP 是一种由肝脏合成的血浆糖蛋白, 在血液循环中与 HDL 结合, 介导胆固醇从 HDL 转 运至极低密度脂蛋白和 LDL 中, 而 CETP 抑制剂 可阻断这一转移过程, 理论上具有抗动脉粥样硬 化和降低心血管风险的作用。研究表明, 尽管 CEPT 抑制剂提高了血浆 HDL-C 水平, 但大多数 CETP 抑制剂 (如托彻普、达塞曲匹、韦特拉比)治 疗无效或出现心血管不良反应 ${ }^{[62-64]}$,药物开发因 此停止。但在一项针对动脉籿样硬化性血管疾病 患者的大型试验中, 另一种 CETP 抑制剂安塞曲匹 在他汀类药物治疗的基础上可减少心血管事件的 发生,但是并未改变乳腺癌等癌症的发病率 ${ }^{[65]}$ 。 目前关于贝特类和 HDL-C 与癌症风险的研究结果 仍存在争议, 尚不能用于临床抗癌治疗。另一种 药物烟酸可使 HDL-C 升高 20\% 25\%, 同时降低三 酰甘油和 LDL-C 水平 ${ }^{[66]}$, 但相关研究结果并未显 示出其对肿瘤具有显著的抑制作用。综上, 基于 HDL 的降血脂药物对人类肿瘤预防和治疗的影响 仍存在争议, 需要进一步的临床前和临床试验。

\section{3 靶向胆固醇酯化的治疗}

阿伐麦布可靶向ACAT-1, 抑制胆固醇酯化,从 而使细胞内游离胆固醇水平升高。在肿瘤细胞 中, 过量游离胆固醇可通过 UPR-CHOP-MAPK 或 p38-MAPK 通路激活未折叠蛋白反应, 引起内质 网应激, 使细胞内多种代谢稳态发生紊乱, 导致细 胞凋亡。研究表明,使用阿伐麦布抑制肿瘤细胞
胆固醇酯化可有效抑制胰腺癌和前列腺癌等肿瘤 的增殖、转移和侵袭 ${ }^{[67]}$ 。因此, 阿伐麦布有望成 为一种抗肿瘤的候选药。

\section{4 展 望}

在肿瘤微环境中, 肿瘤细胞和免疫细胞、脂肪 细胞、内皮细胞和基质细胞等的脂质代谢受到动 态调控并且相互关联, 从而促进肿瘤细胞的生长、 存活、增殖、迁移、侵袭和转移。基于胆固醇及其 代谢产物对肿瘤发生、发展和侵袭的重要影响,人 们对肿瘤细胞中胆固醇代谢的研究越来越深人。 研究表明, 胆固醇的代谢不仅可通过细胞内致癌 信号进行调节, 还可通过肿瘤微环境的输人进行 调节。反过来,胆固醇代谢紊乱也会改变肿瘤细 胞中的致癌信号通路,并通过分泌成分影响邻近 的正常细胞群。这种复杂性提示我们不仅需要研 究肿瘤细胞中的代谢网络, 还需要研究肿瘤微环 境中细胞间的相互联系对肿瘤细胞代谢的影响， 同时研究干扰肿瘤微环境中细胞的胆固醇代谢对 抗肿瘤治疗的作用,尤其是抗肿瘤免疫和抗血管 生成反应。此外,对可被致癌信号诱导和翻译后 修饰的胆固醇相关酶以及胆固醇代谢调节酶结构 的阐明,将有助于确定针对代谢异常调节的特定 干预措施。深人了解肿瘤胆固醇代谢的特异性调 节将提供一种安全性更好的抗肿瘤治疗新策略。

\section{参考文献}

[1] BROWN M S, RADHAKRISHNAN A, GOLDSTEIN J L. Retrospective on cholesterol homeostasis : the central role of scap[J]. Annu Rev Biochem, 2018, 87(1): 783-807. DOI: 10. 1146/annurev-biochem-062917011852.

[2] IKONEN E. Mechanisms for cellular cholesterol transport: defects and human disease $[\mathrm{J}]$. Physiol Rev, 2006,86(4):1237-1261. DOI:10. 1152/physrev. 00022. 2005.

[3] YOSHIOKA Y,SASAKI J,YAMAMOTO M,et al. Quantitation by (1)H-NMR of dolichol, cholesterol and choline-containing lipids in extracts of normal and phathological thyroid tissue[J]. NMR Biomed, 2000, 13(7):377-383. DOI:10. 1002/1099-1492(200011) $13: 7<377$ : : aid-nbm658>3. 0. co;2-e.

[4] LISCUM L, FINER-MOORE J, STROUD R M, et al. Domain structure of 3-hydroxy-3-methylglutaryl coenzyme A reductase, a glycoprotein of the endoplasmic reticulum $[J]$. J Biol Chem, 1985,260(1): 522-530. DOI : 10. 1016/S0021-9258(18)89764-2. 
[5] KO C W, QU J, BLACK D D, et al. Regulation of intestinal lipid metabolism:current concepts and relevance to disease $[\mathrm{J}]$. Nat Rev Gastroenterol Hepatol, 2020,17(3):169-183. DOI:10. 1038/ s41575-019-0250-7.

[6] NELSON J K, KOENIS D S, SCHEIJ S, et al. EEPD1 Is a novel LXR target gene in macrophages which regulates ABCA1 abundance and cholesterol efflux [J]. Arterioscler Thromb Vasc Biol, 2017,37(3): 423-432. DOI: 10. 1161/ATVBAHA. 116. 308434.

[7] MENG Y, HEYBROCK S, NECULAI D, et al. Cholesterol handling in lysosomes and beyond $[\mathrm{J}]$. Trends Cell Biol, 2020,30(6):452-466. DOI:10. 1016/j. tcb. 2020. 02.007.

[8] BROWN M S, GOLDSTEIN J L. A receptor-mediated pathway for cholesterol homeostasis $[\mathrm{J}]$. Science, 1986,232(4746) :34-47. DOI: 10. 1126/science. 351 3311.

[9] MEYER J M, GRAF G A, VAN DER WESTHUYZEN D R. New developments in selective cholesteryl ester uptake[J]. Curr Opin Lipidology, 2013,24(5): 386-392. DOI: 10. 1097/MOL. 0b013e3283638042.

[10] DAS A, BROWN M S, ANDERSON D D, et al. Three pools of plasma membrane cholesterol and their relation to cholesterol homeostasis $[\mathrm{J} / \mathrm{OL}]$. eLife, 2014,3 : e02882. DOI: 10. 7554/eLife. 02882.

[11] GOLDSTEIN J L, BROWN M S. The LDL receptor [J]. ATVB, 2009,29(4):431-438. DOI:10.1161/ ATVBAHA. 108. 179564.

[12] LUO J, YANG H, SONG B L. Mechanisms and regulation of cholesterol homeostasis $[\mathrm{J}]$. Nat Rev Mol Cell Biol, 2020,21(4):225-245. DOI: 10 . 1038/s41580-019-0190-7.

[13] GLASS C, PITTMAN R C, CIVEN M, et al. Uptake of high-density lipoprotein-associated apoprotein A-I and cholesterol esters by 16 tissues of the rat in vivo and by adrenal cells and hepatocytes in vitro $[\mathrm{J}]$. J Biol Chem, 1985,260(2) : 744-750. DOI : 10. 1016/S00219258(20)71160-9.

[14] GLASS C, PITTMAN R C, WEINSTEIN D B, et al. Dissociation of tissue uptake of cholesterol ester from that of apoprotein A-I of rat plasma high density lipoprotein:selective delivery of cholesterol ester to liver, adrenal, and gonad $[J]$. Proc Natl Acad Sci USA, 1983,80(17) :5435-5439. DOI: 10. 1073/pnas. 80. 17. 5435.

[15] NECULAI D, SCHWAKE M, RAVICHANDRAN M, et al. Structure of LIMP-2 provides functional insights with implications for SR-BI and CD36[J]. Nature, 2013,504(7478):172-176. DOI:10. 1038/nature 12684.

[16] HAMPTON R Y. A cholesterol toggle switch $[\mathrm{J}]$. Cell Metab, 2008,8(6):451-453. DOI:10. 1016/j. cmet.
2008. 11. 006.

[17] HORTON J D, SHAH N A, WARRINGTON J A, et al. Combined analysis of oligonucleotide microarray data from transgenic and knockout mice identifies direct SREBP target genes[J]. Proc Natl Acad Sci USA, 2003,100(21):12027-12032. DOI:10.1073/pnas. 1534923100 .

[18] GOLDSTEIN J L, DEBOSE-BOYD R A, BROWN M S. Protein sensors for membrane sterols $[\mathrm{J}]$. Cell, 2006,124(1):35-46. DOI: 10. 1016/j. cell. 2005. 12. 022.

[19] BOVEngA F, SABBÀ C, MOSChETTA A. Uncoupling nuclear receptor LXR and cholesterol metabolism in cancer[J]. Cell Metab, 2015,21(4): 517-526. DOI: 10. 1016/j. cmet. 2015. 03. 002.

[20] LI C S, WU W, XIE K K, et al. HMGCR is upregulated in gastric cancer and promotes the growth and migration of the cancer cells $[\mathrm{J}]$. Gene, 2016,587 (1) :42-47. DOI: 10. 1016/j. gene. 2016. 04. 029.

[21] QIU Z, YUAN W, CHEN T, et al. HMGCR positively regulated the growth and migration of glioblastoma cells $[\mathrm{J}]$. Gene, 2016,576(1):22-27. DOI:10. 1016/ j. gene. 2015. 09. 067.

[22] ASHIDA S, KAWADA C, INOUE K. Stromal regulation of prostate cancer cell growth by mevalonate pathway enzymes HMGCS1 and HMGCR [J]. Oncol Lett, 2017,14(6) :6533-6542. DOI : 10. 3892/ol. 2017. 7025.

[23] KONG Y, CHENG L, MAO F, et al. Inhibition of cholesterol biosynthesis overcomes enzalutamide resistance in castration-resistant prostate cancer (CRPC) [J]. J Biol Chem, 2018,293(37) :1432814341. DOI: 10. 1074/jbc. RA118. 004442.

[24] LEE J S, ROBERTS A, JUAREZ D, et al. Statins enhance efficacy of venetoclax in blood cancers $[\mathrm{J}]$. Sci Transl Med, 2018,10(445): eaaq1240. DOI: 10. 1126/scitranslmed. aaq1240.

[25] YANG J, WANG L, JIA R. Role of de novo cholesterol synthesis enzymes in cancer[J]. J Cancer, 2020,11 (7): 1761-1767. DOI: 10. 7150/jca. 38598.

[26] BROWN D N, CAFFA I, CIRMENA G, et al. Squalene epoxidase is a bona fide oncogene by amplification with clinical relevance in breast cancer [J]. Sci Rep, 2016,6(1) : 19435. DOI:10. 1038/ srep19435.

[27] CIRMENA G, FRANCESCHELLI P, ISNALDI E, et al. Squalene epoxidase as a promising metabolic target in cancer treatment $[\mathrm{J}]$. Cancer Lett, 2018,425:13-20. DOI : 10. 1016/j. canlet. 2018. 03. 034.

[28 ] LIU D, WONG C C, FU L, et al. Squalene epoxidase drives NAFLD-induced hepatocellular carcinoma and is a pharmaceutical target $[\mathrm{J} / \mathrm{OL}]$. Sci Transl Med, 2018,10(437) : eaap9840. DOI: 10. 1126/scitranslm 
ed. aap9840.

[29] ZHOU T, ZHAN J, FANG W, et al. Serum low-density lipoprotein and low-density lipoprotein expression level at diagnosis are favorable prognostic factors in patients with small-cell lung cancer (SCLC) $[\mathrm{J}]$. BMC Cancer, 2017,17(1):269. DOI:10.1186/ s12885-017-3239-z.

[30 ] GALLAGHER E J, ZELENKO Z, NEEL B A, et al. Elevated tumor LDLR expression accelerates LDL cholesterol-mediated breast cancer growth in mouse models of hyperlipidemia[J]. Oncogene, 2017,36 (46) :6462-6471. DOI: 10. 1038/onc. 2017. 247.

[31] GUILLAUMOND F, BIDAUT G, OUAISSI M, et al. Cholesterol uptake disruption, in association with chemotherapy, is a promising combined metabolic therapy for pancreatic adenocarcinoma $[\mathrm{J}]$. Proc Natl Acad Sci USA, 2015,112(8):2473-2478. DOI: $10.1073 /$ pnas. 1421601112.

[32] GUO D, REINITZ F, YOUSSEF M, et al. An LXR agonist promotes glioblastoma cell death through inhibition of an EGFR/AKT/SREBP-1/LDLRdependent pathway $[\mathrm{J}]$. Cancer Discov, 2011,1(5): 442-456. DOI: 10. 1158/2159-8290. CD-11-0102.

[33] YUE S, LI J, LEE S Y, et al. Cholesteryl ester accumulation induced by PTEN loss and PI3K/AKT activation underlies human prostate cancer aggressiveness $[J]$. Cell Metab, 2014,19(3):393-406. DOI: $10.1016 /$ j. cmet. 2014. 01. 019.

[34] MENARD J A, CHRISTIANSON H C, KUCHARZEWSKA P, et al. Metastasis stimulation by hypoxia and acidosis-induced extracellular lipid uptake is mediated by proteoglycan-dependent endocytosis [J]. Cancer Res, 2016,76(16):4828-4840. DOI: 10. 1158/0008-5472. CAN-15-2831.

[35] ZELCER N, HONG C, BOYADJIAN R, et al. LXR regulates cholesterol uptake through Idol-dependent ubiquitination of the LDL receptor $[J]$. Science, 2009,325(5936):100-104. DOI:10. 1126/science. 1168974.

[36] AYLON Y, OREN M. The Hippo pathway,p53 and cholesterol $[\mathrm{J}]$. Cell Cycle, 2016,15(17):22482255. DOI: $10.1080 / 15384101.2016 .1207840$.

[37] XU D, WANG Z, XIA Y, et al. The gluconeogenic enzyme PCK1 phosphorylates INSIG1/2 for lipogenesis [J]. Nature, 2020,580(7804):530-535. DOI: $10.1038 / \mathrm{s} 41586-020-2183-2$.

[38 ] OHTAKI S, WANIBUCHI M, KATAOKA-SASAKI Y, et al. ACTC1 as an invasion and prognosis marker in glioma $[J]$. JNS, 2017,126(2):467-475. DOI: 10 . 3171/2016. 1. JNS152075.

[39] IKONEN E. Cellular cholesterol trafficking and compartmentalization[J]. Nat Rev Mol Cell Biol, 2008,9(2) :125-138. DOI: 10. 1038/nrm2336.
[40] LIN C Y, GUSTAFSSON J Å. Targeting liver X receptors in cancer therapeutics $[J]$. Nat Rev Cancer, 2015,15(4):216-224. DOI:10.1038/ nrc3912.

[41] TAO R, XIONG X, DEPINHO R A, et al. Hepatic SREBP-2 and cholesterol biosynthesis are regulated by FoxO3 and Sirt6[J]. J Lipid Res, 2013,54 (10):2745-2753. DOI:10. 1194/jlr. M039339.

[42] FLAVENY C A, GRIFFETT K, EL-GENDY B E D M, et al. Broad anti-tumor activity of a small molecule that selectively targets the warburg effect and lipogenesis $[\mathrm{J}]$. Cancer Cell, 2015,28(1):42-56. DOI : $10.1016 /$ j. ccell. 2015. 05. 007.

[43] RIOLS F, BERTRAND-MICHEL J. Analysis of oxysterols $[\mathrm{J}]$. Methods Mol Biol, 2018, 1730:267275. DOI:10. 1007/978-1-4939-7592-1_19.

[44] CHEN J, YE Y, LIU P, et al. Suppression of T cells by myeloid-derived suppressor cells in cancer $[\mathrm{J}]$. Human Immunol, 2017,78(2):113-119. DOI: 10 . 1016/j. humimm. 2016. 12. 001.

[45] SAXENA K, SHIPLEY G G. Structural studies of detergent-solubilized and vesicle-reconstituted lowdensity lipoprotein (LDL) receptor $[\mathrm{J}]$. Biochemistry, 1997,36(50):15940-15948. DOI:10. 1021/ bi971579p.

[46] SIVAPRASAD U, ABBAS T, DUTTA A. Differential efficacy of 3-hydroxy-3-methylglutaryl CoA reductase inhibitors on the cell cycle of prostate cancer cells $[\mathrm{J}]$. Mol Cancer Ther, 2006,5(9):2310-2316. DOI : 10. 1158/1535-7163. MCT-06-0175.

[47] SONG X, LIU B C, LU X Y, et al. Lovastatin inhibits human $\mathrm{B}$ lymphoma cell proliferation by reducing intracellular ROS and TRPC6 expression[J]. BBAMol Cell Res, 2014,1843(5):894-901. DOI: 10 . 1016/j. bbamcr. 2014. 02. 002.

[48] TU Y S, KANG X L, ZHOU J G, et al. Involvement of Chk1-Cdc25A-cyclin A/CDk2 pathway in simvastatin induced S-phase cell cycle arrest and apoptosis in multiple myeloma cells $[\mathrm{J}]$. Eur J Pharmacol, 2011,670(2-3):356-364. DOI: 10. 1016/j. ejphar. 2011. 09. 031.

[49] MENTER D G, RAMSAUER V P, HARIRFOROOSH $\mathrm{S}$, et al. Differential effects of pravastatin and simvastatin on the growth of tumor cells from different organ sites [J/OL]. PLoS One, 2011,6(12): e28813. DOI: 10. 1371/journal. pone. 0028813.

[50] XIA Y, XIE Y, YU Z, et al. The Mevalonate pathway is a druggable target for vaccine adjuvant discovery [J/OL]. Cell, 2018,175(4) : 1059-1073. e21. DOI : 10. 1016/j. cell. 2018. 08. 070.

[51] ABDEL-RAHMAN O. Statin treatment and outcomes of metastatic pancreatic cancer:a pooled analysis of two phase III studies $[\mathrm{J}]$. Clin Transl Oncol, 
2019,21(6):810-816. DOI: 10. 1007/s12094-0181992-3.

[52] BRÅNVALL E, EKBERG S, ELORANTA S, et al. Statin use is associated with improved survival in multiple myeloma:A Swedish population-based study of 4315 patients $[\mathrm{J}]$. Am J Hematol, 2020,95 (6) : 652-661. DOI: 10. 1002/ajh. 25778.

[53] CARDWELL C R, HICKS B M, HUGHES C, et al. Statin use after colorectal cancer diagnosis and survival:a population-based cohort study $[\mathrm{J}]$. JCO, 2014,32(28):3177-3183. DOI: 10. 1200/JCO. 2013. 54. 4569.

[54] HAN J Y, LEE S H, YOO N J, et al. A randomized phase II study of gefitinib plus simvastatin versus gefitinib alone in previously treated patients with advanced non-small cell lung cancer $[\mathrm{J}]$. Clin Cancer Res, 2011,17(6):1553-1560. DOI: 10 . 1158/1078-0432. CCR-10-2525.

[55 ] LEE Y, LEE K H, LEE G K, et al. Randomized phase study of afatinib plus simvastatin versus afatinib alone in previously treated patients with advanced nonadenocarcinomatous non-small cell lung cancer [J]. Cancer Res Treat, 2017,49(4):1001-1011. DOI: $10.4143 /$ crt. 2016. 546.

[56] DUNCAN R E, EL-SOHEMY A, ARCHER M C. Statins and the risk of cancer $[J]$. JAMA, 2006,295 (23) :2720. DOI: 10. 1001/jama. 295. 23. 2720-a.

[57] KIM S T, KANG J H, LEE J, et al. Simvastatin plus capecitabine-cisplatin versus placebo plus capecitabine-cisplatin in patients with previously untreated advanced gastric cancer: A double-blind randomised phase 3 study $[J]$. Eur J Cancer, 2014, 50(16): 2822-2830. DOI:10. 1016/j. ejca. 2014. 08. 005.

[58] FARMER J A. Intensive lipid lowering with simvastatin and ezetimibe in aortic stenosis (the SEAS trial) $[J]$. Curr Atheroscler Rep, 2009,11 (2) : 82-83.

[59] JOSEPH L, ROBINSON J G. Proprotein convertase subtilisin/kexin type 9 (PCSK9) inhibition and the future of lipid lowering therapy $[\mathrm{J}]$. Prog Cardiovascular Dis, 2015,58(1): 19-31. DOI: 10. 1016/j. pcad. 2015. 04. 004.

[60] NOWAK C, ÄRNLÖV J. A Mendelian randomization study of the effects of blood lipids on breast cancer risk [J]. Nat Commun, 2018,9(1):3957. DOI: 10 . 1038/s41467-018-06467-9.

[61 ] REVILLA G, CEDÓ L, TONDO M, et al. LDL,HDL and endocrine-related cancer:From pathogenic mechanisms to therapies $[\mathrm{J} / \mathrm{OL}]$. Seminars Cancer Biol, 2020. DOI: 10. 1016/j. semcancer. 2020. 11. 012.

[62 ] LINCOFF A M, NICHOLLS S J, RIESMEYER J S, et al. Evacetrapib and cardiovascular outcomes in highrisk vascular disease[J]. N Engl J Med, 2017,376 (20):1933-1942. DOI:10. 1056/NEJMoa1609581.

[63] SCHWARTZ G G, OLSSON A G, ABT M, et al. Effects of dalcetrapib in patients with a recent acute coronary syndrome $[J]$. N Engl J Med, 2012,367 (22):2089-2099. DOI:10. 1056/NEJMoa1206797.

[64] BARTER P J, CAULFIELD M, ERIKSSON M, et al. Effects of torcetrapib in patients at high risk for coronary events $[\mathrm{J}]$. N Engl J Med, 2007,357 (21 ):2109-2122. DOI:10. 1056/NEJMoa0706628.

[65] BOWMAN L, HOPEWELL J C, CHEN F, et al. HPS3/TIMI55-REVEAL collaborative group effects of anacetrapib in patients with atherosclerotic vascular disease $[J]$. N Engl J Med, 2017,377(13) : 12171227. DOI: $10.1056 /$ NEJMoa1706444.

[66] KAMANNA S V, KASHYAP L M. Mechanism of action of niacin[J]. Am J Cardiology, 2008,101 (8) : S20-S26. DOI: 10. 1016/j. amjcard. 2008. 02. 029.

[67 ] LI J, GU D, LEE S S Y, et al. Abrogating cholesterol esterification suppresses growth and metastasis of pancreatic cancer $[\mathrm{J}]$. Oncogene, 2016,35(50): 6378-6388. DOI:10. 1038/onc. 2016. 168.

[本文编辑 沈 敏 余 方] 